\title{
Determination of an effective pore dimension for microporous media
}

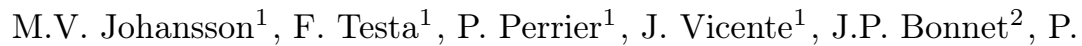 \\ Moulin $^{2}$, I. Graur ${ }^{1}$ \\ ${ }^{1}$ Aix-Marseille Université, CNRS, IUSTI UMR 7343, 5 rue E. Fermi, 13453, \\ Marseille,France \\ ${ }^{2}$ Aix Marseille Université, CNRS, Centrale Marseille, M2P2 UMR 7340, Equipe Procédés \\ Membranaires (EPM), Europôle de l'Arbois, BP80, Pavillon Laennec, Hall C, 13545 Aix en \\ Provence Cedex, France
}

\begin{abstract}
The transient method of the mass flow rate and permeability measurements through a microporous media, developed previously, is used here to extract different characteristics of the media. By implementing the model of porous media as a bundle of capillaries the effective pore dimension is extracted from the measurements, and its physical interpretation is given. This methodology shows promising results to be used as a non-destructive method of micro-andnanoporous media analysis. The permeability is also extracted directly from the measurements of the pressure variation in time. By using additional information about the sample porosity, the number of capillaries, the tortuosity and the internal surface of the sample are calculated. The extracted values are very close to that obtained by the mercury porosimetry and by microtomography.
\end{abstract}

\section{Introduction}

The determination of characteristics of porous media permeability like the micro and nanoporous membranes or ultra-tight shale-gas reservoirs is still a challenge up to now. The low porous media find a broad application in medicine [1], biotechnology for separation and filtration [2]. The recent development of porous ceramic media with high thermal, chemical and structural stability and the ability to have catalytic properties has opened up new horizons for membranes applications, for example, in high-temperature gas separation and catalytic reactions [3]. Unconventional resources, such as ultra-tight shale-gas reservoirs of very small pores (in nanoscale) play a significant role in securing hydrocarbon energy because of their potential to offset declines in conventional gas production [4]. The morphology of the porous structure dominates the fluid flow through a porous medium. Therefore, it is important to characterize the geometrical properties of a porous medium quantitatively. Different methods exist for the measurements of the average pore size and pore size distribution.

Email addresses: martin.viktor.johansson@gmail.com (M.V. Johansson $\left.{ }^{1}\right)$,

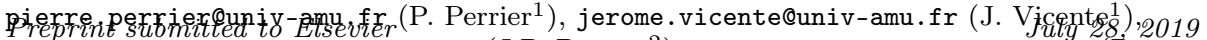
jean-philippe.bonnet@univ-amu.fr (J.P. Bonnet $\left.{ }^{2}\right)$, philippe.moulin@univ-amu.fr (P. Moulin $\left.{ }^{2}\right)$, irina.martin@univ-amu.fr (I. Graur ${ }^{1}$ ) 
The choice of the most appropriate method depends on the application of the porous solid, its chemical and physical nature and the range of pore size. The most commonly used methods are [5]: mercury porosimetry, where the pores are filled with mercury under pressure. This method is suitable for many materials with pores in the appropriate diameter range from $0.003 \mu \mathrm{m}$ to $300 \mu \mathrm{m}$. From mesopore to micropore size analysis, BET method [6], can be done by gas adsorption, usually nitrogen, at liquid nitrogen temperature. This method can be used for pores in the approximate diameter range from $1 \mathrm{~nm}$ to $0.1 \mu \mathrm{m}$. The pore size diameter can also be determined via direct observation methods: scanning electron microscopy (SEM), field-emission scanning electron microscopy (FESEM), environmental scanning electron microscopy (ESEM), and atomic force microscopy (AFM), [7], [8]. The tomography analysis of a porous structure can allow the determination of the internal structure of a sample limited by the characteristics of their spatial resolution [9]. All these methods require either preliminary sample preparation or lead to the complete sample destruction, furthermore, they only use a small part of the sample for analysis.

We propose here a simple approach for the non-destructive porous sample characterization by measuring the pressure variation in the inlet and outlet tanks (or just the pressure difference between them). The experimental methodology, based on the constant volume technique, was initially developed for the isothermal and non-isothermal measurements of the mass flow rate through the microchannels [10] and has been recently adapted for the analysis of porous samples [11]. The gas permeability of the porous sample can be easily obtained directly from the pressure evolution in time without calculation of the mass flow rate.

The measurements are analyzed by assuming the porous media have similar behavior as the classical bundle of capillaries model, first suggested by Kozeny [12] and then extended by Carman [13] to allow for torturous capillaries. In our analysis we assume that the capillary tubes have the same radius. This allows us to find an unique parameter (capillary's radius) to characterize the porous structure. This unique parameter helps also to determine the gas flow regime, by introducing the Knudsen number as the ratio of the molecular mean free path and the capillary radius, and then by referring on this Knudsen number to distinguish the flow regimes. Recently, the models of a bundle of capillary tubes of variable shape and size cross-section were developed, [14], [15], but all the models were used either for the liquid or for two phase flows, which physics is different from the single phase flows.

The model of a bundle of capillaries with gas flow inside was considerably improved by Klinkenberg [16] taking into account the slip flow regime through the capillaries. In the present article, from the measured mass flow rate the effective pore size is estimated by using the fitting procedure via slip flow expression. The obtained effective pore sizes are then compared to mercury porosimetry and micro-computed tomography $(\mu \mathrm{CT})$ results. The proposed technique of the effective pore size measurement can be used as a non-destructive method for quality verification. Furthermore, this method is independent of the exterior sample geometry. When the effective pore size is known and by using the infor- 
mation about porosity the permeability, apparent permeability, and tortuosity coefficients as well as the surface-to-volume ratio can be easily obtained.

\section{Experimental methodology}

The experimental methodology, applied in this article, is described in details in Ref. [11]. We present here only the summary of this technique, essential to understand the data treatment. From measurements of pressure variation over time we calculate the important characteristics of porous media such as mass flow rate and permeability, and then effective pore size dimension.

\subsection{Experimental apparatus}

The experimental setup is a high vacuum system capable of measuring up to 5 decades of pressure. In the presented experiment the mean pressure is varied from $75 \mathrm{~Pa}$ up to $131 \mathrm{kPa}$. This large pressure measurement range is achieved by using three pairings of four Capacitance Diaphragm Manometers (CDM) with full-scale $\left(\mathrm{CDM}_{1}-\mathrm{CDM}_{2}\right): 133 \mathrm{kPa}-133 \mathrm{kPa}, 133 \mathrm{kPa}-13.3 \mathrm{kPa}$ and $13.3 \mathrm{kPa}$ $1.33 \mathrm{kPa}$. Four high purity gas bottles with test gases, Helium, Neon, Nitrogen, Argon (Air Liquide, France) are used. The pumping is performed by a twostage Diaphragm Vacuum Pump (DVP) and a Turbomolecular Pump (TMP). Each side of the porous medium is connected to two reservoirs, of volumes $V_{1}$ and $V_{2}$, for the high and low-pressure, respectively. Both tanks volumes, including the volumes of the valves, connecting tubes and pressure sensors, are measured accurately, and these volumes are equal to $V_{1}=255.8 \pm 5.5 \mathrm{~cm}^{3}$ and $V_{2}=238.8 \pm 5.1 \mathrm{~cm}^{3}$, for the high and low pressure tanks, respectively. The reservoirs are connected only by a porous sample which is fixed with a vacuum glue.

Two microporous samples, used in the experiments and mentioned in the following as the first and second discs, have a cylindrical shape (disc) with the same radius and thickness (in main flow direction) equal to $4.75 \pm 0.01 \mathrm{~mm}$ and $L=2.3 \pm 0.01 \mathrm{~mm}$, respectively. The characteristics of these microporous discs are the same as of the ceramic membranes used in micro-to-nano filtration. For such ceramic microporous media, depending on manufacturer, porosity is in the range $15 \%-30 \%$ with pore diameter ranging from $1 \mu \mathrm{m}$ to $10 \mu \mathrm{m}$. The total volume of each porous disc is $0.14 \mathrm{~cm}^{3}$, so by taking $30 \%$ of porosity a gas volume inside the medium is approximately $0.042 \mathrm{~cm}^{3}$, which is much smaller than the volume of each tank.

The experiments are performed within a narrow temperature range (around room temperature, $29-31^{\circ} \mathrm{C}$ ), excluding any heat source in an environment. The temperature is measured using the thermocouple with the accuracy of 0.6 $\mathrm{K}$.

\subsection{Mass flow rate measurements}

The constant volume technique [17], [18] and the methodology, analogous to the pulse decay method [19], [11], are implemented here to measure the 
113

mass flow rate through samples of a microporous ceramic medium. The applied experimental technique allows us to deduce the mass flow rate and also the permeability from the pressure variation in time in both tanks. This approach needs to have a stable temperature during the measurements, see discussion in Ref. [11]. Therefore, if the temperature variations during the experimental time are small compared to the pressure variations we can calculate the mass flow rate from the pressure variation in each tank as following:

$$
\dot{M}_{1}=-\frac{\mathrm{d} M_{1}}{\mathrm{~d} t}=-\frac{V_{1}}{\mathcal{R} T} \frac{\mathrm{d} p_{1}}{\mathrm{~d} t}, \quad \dot{M}_{2}=\frac{\mathrm{d} M_{2}}{\mathrm{~d} t}=\frac{V_{2}}{\mathcal{R} T} \frac{\mathrm{d} p_{2}}{\mathrm{~d} t} .
$$

Here $\dot{M}_{i}$ and $p_{i}, i=1,2$ are the mass flow rate and pressure in the tank $i$, respectively, $\mathcal{R}$ is the specific gas constant, $T$ is the gas temperature, the same in each tank, $t$ is the time. Equations (1) are obtained with an assumption that the gas follows the ideal gas law. In the present study, the maximal considered pressure is slightly above atmospheric pressure (up to $131 \mathrm{kPa}$ ); therefore we do not consider here the real gas effects. However, the proposed approach can be generalized to take into account the real gas effects by using, for example, the van der Waals equation instead of the ideal gas law. The estimations of the compressibility factor under our experimental conditions are provided in AppendixA.

The ideal gas law is valid under equilibrium condition; however, the gas pressure and gas mass in a tank change in time. Here we assume that we have a quasi-steady process, that is, we have a succession of local equilibrium. This assumption is true when we have a small unbalancing force which modifies the system slower than the system reaches a local equilibrium, see AppendixB for a further discussion.

Very often it is convenient to express the mass flow rate in function of the pressure difference, $\Delta p(t)=p_{1}(t)-p_{2}(t)$, between two tanks:

$$
\dot{M}(t)=-\frac{V_{0}}{\mathcal{R} T} \frac{\mathrm{d}(\Delta p(t))}{\mathrm{d} t}, \quad V_{0}=\frac{V_{1} V_{2}}{V_{1}+V_{2}},
$$

where $V_{0}$ is the effective volume. It is clear that the mass flow rate can be calculated using expressions (1) and (2), when the pressure variation in each tank or the pressure difference between them in time is known. To measure the mass flow rate, first, the initial pressure difference is settled between the tanks, then the gas starts to flow from higher to lower pressure tank up to the same final pressure $p_{\mathrm{f}}$ is reached, see Fig. 2 in [11]. During the experiments the pressure variations over time in each tank are recorded, then, their difference is fitted by using the exponential fitting function [19], [11]:

$$
\Delta p(t)=\Delta p_{0} \exp \left(-\left(t-t_{0}\right) / \tau\right),
$$

where $\tau$ is the pressure relaxation time, $\Delta p_{0}$ is the initial pressure difference between the tanks at time $t=t_{0}$. Similar exponential representations of the pressure evolution over time in the first, $p_{1}(t)$, and second, $p_{2}(t)$, tank can be also written in a form similar to Eq. (3), see Refs. [19], [11]. The pressure 
evolution in time in each tank and the pressure difference between two tanks as a function of time and their corresponding fitting functions are shown in Fig. 2 (a) and (b), respectively.

By using Eq. (2) for the mass flow rate, the exponential representation of the pressure difference in time, Eq. (3), and its analytical derivative, we can now express the mass flow rate as

$$
\dot{M}(t)=-\frac{V_{0}}{\mathcal{R} T} \frac{\mathrm{d}(\Delta p(t))}{\mathrm{d} t}=\frac{V_{0}}{\mathcal{R} T} \frac{\Delta p_{0}}{\tau} \exp \left(-\frac{t-t_{0}}{\tau}\right) .
$$

From Eqs. (1) we can also express the mass flow rate using the analogous to Eq. (3) exponential representation of the pressure variation over time in each tank, see Refs. [19], [11]. To obtain the mass flow rate from the pressure variation measurements, the pressure variation in time, $\tau$, is fitted using pressure relaxation time as a single fitting parameter, then the mass flow rate can be calculated from Eq. (4).

The classical uncertainty calculation technique is used to estimate the measurement uncertainty of the mass flow rate, which for our experimental conditions lies in the range $3.6-5.1 \%$, see Ref. [11] for more details.

\subsection{Gas permeability measurements}

The Darcy law [20] allows us to relate the instantaneous discharge (or volumetric) flow rate through a porous medium, $Q$, to the pressure drop over a given distance $L$, which is the thickness of a porous sample (disc):

$$
Q=\frac{K S}{\mu} \frac{\Delta p}{L}
$$

where $K$ is the permeability, $S$ is the cross-section of the porous sample, $\mu$ is the viscosity, which is calculated as [21]:

$$
\mu=\mu_{\text {ref }}\left(\frac{T}{T_{\text {ref }}}\right)^{\omega},
$$

171 where $\omega$ is the gas viscosity index, $\mu_{\text {ref }}$ is the gas viscosity at temperature $T_{\text {ref }}=273.15 \mathrm{~K}[21]$, see also Table 1.

\begin{tabular}{|c|c|c|c|c|}
\hline Gas & $\mu_{\text {ref }} \times 10^{-5}[\mathrm{~Pa} \cdot \mathrm{s}]$ & $\omega$ & $\mathcal{R}\left[\mathrm{J} \cdot \mathrm{kg}^{-1} \cdot \mathrm{K}^{-1}\right]$ & Molar mass $\mathcal{M}\left[\mathrm{g} \cdot \mathrm{mol}^{-1}\right]$ \\
\hline $\mathrm{He}$ & 1.865 & 0.66 & 2077.1 & 4.003 \\
$\mathrm{Ne}$ & 2.976 & 0.66 & 412.02 & 20.18 \\
$N_{2}$ & 1.656 & 0.74 & 296.80 & 28.00 \\
$\mathrm{Ar}$ & 2.117 & 0.81 & 208.13 & 39.95 \\
\hline
\end{tabular}

Table 1: Useful characteristics of the gases [21] used in present experiments

The volumetric flow rate, used in Eq. (5), is related to the mass flow rate and the gas density $\rho$ as:

$$
Q=\frac{\dot{M}}{\rho}=\dot{M} \frac{\mathcal{R} T}{p}
$$


then by integrating along the porous sample and by using the mass conservation property, we obtain the expression, analogous to Eq. (5), which relates the mass flow rate, instead of volumetric flow rate, to the permeability

$$
\dot{M}=\frac{K S}{\mu} \frac{\Delta p}{L} \frac{p_{\mathrm{m}}}{\mathcal{R} T} .
$$

Here $p_{\mathrm{m}}$ is the mean pressure between two tanks, $p_{\mathrm{m}}=0.5\left(p_{1}+p_{2}\right)$. Then, using the expression of the mass flow rate via the pressure variation in tanks, Eq. (2), and following the technique developed in Ref. [11] we can relate permeability to the pressure difference variations between the tanks and finally obtain the expression of the gas permeability through the fitting parameter, $\tau$, pressure relaxation time, see Ref. [11] for more details:

$$
K=\frac{\mu}{\tau p_{\mathrm{m}}} \frac{L V_{0}}{S} .
$$

It is worth to note that the previous expression is obtained under the condition of the mean pressure constancy during an experimental run. This condition is satisfied, when the tanks volumes are equal, $V_{1}=V_{2}$. For the case of different tanks volumes $V_{1} \neq V_{2}$, the analytical expression was derived in [11]. This expression relates the variation of the mean pressure during the experimental time to the tanks volumes ratio, $V_{1} / V_{2}$, and the initial pressure ratio, $p_{1}\left(t_{0}\right) / p_{2}\left(t_{0}\right)$, between the tanks.

The uncertainty of the permeability measurements, when using Eq. (9), is calculated by the classical way, similar to the calculation of the uncertainty on the mass flow rate. Under our experimental conditions the measurement uncertainty on the permeability lies in the range $5.0-6.4 \%$.

\section{Modeling of the porous structure}

Different type of modeling can be used to characterize the flow through microporous media. One of the simplest and, in the same time, efficient models of a microporous medium is its representation as a bundle of several numbers of capillaries with the circular cross-section of the same or different diameters [12], [20]. All the capillaries (pores) can be parallel and have a length $L_{c}$ equal to the length $L$ (thickness) of the porous medium, see Fig. 1 (left). However, in the real samples, this capillary length can be different from the length of the porous medium because of the random orientation of the capillaries. As a result, the capillary length is generally longer than the medium thickness, see Fig. 1 (right). To account for this fact a tortuosity $l_{\tau}[22]$ is introduced as

$$
l_{\tau}=\frac{L_{c}}{L} .
$$

The sample porosity $\varepsilon$ is defined as

$$
\varepsilon=\frac{V_{c}}{V},
$$


where $V_{c}$ is the volume of void-space (such as fluids) and $V$ is the total or bulk volume of a solid material.

If the porous medium is represented as a bundle of $N$ capillaries of the same radius $a$ and of the length $L_{c}$, different from the membrane thickness $L$, Eq. (10), the porosity is calculated as:

$$
\varepsilon=\frac{N \pi a^{2} L_{c}}{S L}=\frac{N \pi a^{2} l_{\tau}}{S} .
$$

With the same set of parameters a very useful characteristic of porous medium can be calculated, the Specific Surface Area (SSA) defined as the the ratio of the internal surface to the sample volume

$$
\mathcal{S}_{\mathrm{A}}=\frac{2 \pi a L_{c} N}{S L}=\frac{2 \pi a N l_{\tau}}{S} \quad\left[\frac{\mathrm{m}^{2}}{\mathrm{~m}^{3}}\right] .
$$

Finally the used here model of the porous medium has 4 unknown parameters. Three among them, the parameters $a, N$ and $l_{\tau}$ (or $\varepsilon$ ) are defined above. The forth parameter, the velocity slip coefficient, $\sigma_{p}$, (or the accommodation coefficient, $\alpha$ ), are presented in Section 4.2, where their physical meaning and the typical values are given. In the following, we will show how these characteristics of a porous medium can be extracted when using the presented above model of a bundle of capillaries.

\section{Expressions of the mass flow rate through a single capillary}

\subsection{Flow regimes}

The microporous medium is modeled here as a bundle of capillaries, so it is worth to define first different possible flow regimes in a capillary and to present then the expressions of the mass flow rate through a capillary for these flow regimes. Usually the flow regimes could be identified through the Knudsen number, which is calculated as the ratio between the equivalent molecular mean free path $\ell$ and the characteristic dimension $a$ of the capillary (its radius):

$$
K n=\frac{\ell}{a} .
$$

The equivalent molecular mean free path $\ell$ is defined as

$$
\ell=\frac{\mu v_{0}}{p_{m}},
$$

where $v_{0}$ is the most probable molecular speed

$$
v_{0}=\sqrt{2 \mathcal{R} T} .
$$

It is convenient to introduce also the rarefaction parameter $\delta$ which is directly proportional to the pressure and related to the Knudsen number as

$$
\delta=\frac{a}{\ell}=\frac{1}{K n} .
$$


We adopt here the classical definition of gas flow regimes in terms of the Knudsen number or rarefaction parameter [23]: continuum flow regime $(K n<0.01$ or $\delta>100)$; slip flow regime $(0.01<K n<0.1$ or $10<\delta<100)$; transitional flow regime $(0.1<K n<10$ or $0.1<\delta<10)$; free molecular flow regime $(K n>10$ or $\delta<0.1)$.

\subsection{Mass flow rate expressions for a single capillary}

In the case of the slip flow regime $(10 \leq \delta \leq 100)$ the mass flow rate through a tube (capillary) of a radius $a$ can be obtained from the Stokes equation

$$
\frac{\mu}{r}\left(\frac{\partial}{\partial r}\left(r \frac{\partial u}{\partial r}\right)\right)=\frac{\mathrm{d} p}{\mathrm{~d} z}
$$

subjected to the velocity slip boundary condition at the solid surface

$$
u=\left.\sigma_{p} \ell \frac{\partial u}{\partial r}\right|_{r=a} .
$$

In the previous relations $u$ is the longitudinal flow velocity, $z$ is the longitudinal flow direction, $p$ is the local gas pressure, $\sigma_{p}$ is the velocity slip coefficient, which depends on the type of the gas-surface interaction. Using the kinetic theory the velocity slip coefficient was calculated in Ref. [23] to be equal to 1.018 in the case of diffuse gas-surface interaction. The accommodation coefficient, $\alpha$, characterizes also the gas-surface interaction: it is equal to 1 for the case of diffuse interaction (complete accommodation) in the frame of Maxwellian scattering kernel [24]. Both coefficients are related between them: the authors of Refs. [25], [26] suggested to use the following relation:

$$
\sigma_{p}(\alpha)=\frac{2-\alpha}{\alpha}\left(\sigma_{p}(\alpha=1)-0.1211(1-\alpha)\right) .
$$

By integrating Eq. (18) with the boundary condition (19) and the symmetry condition on the tube axis we obtain the velocity profile over the capillary crosssection. Next, by integrating this velocity profile and then, by integrating along the tube (according to $z$ variable) from 0 to the capillary length $L_{c}$ and using the mass conservation property we obtain finally the mass flow rate through a single tube (capillary) for the slip flow regime:

$$
\dot{M}=\dot{M}_{\mathrm{P}}\left(\frac{1}{4}+\frac{\sigma_{p}}{\delta}\right)
$$

where $\dot{M}_{\mathrm{P}}$ is the classical Poiseuille masse flow rate through a capillary of a radius $a$ and the length $L_{c}$ in the hydrodynamic flow regime $(\delta \geq 100)$ :

$$
\dot{M}_{\mathrm{P}}=\frac{\pi a^{4}}{L_{c}} \frac{\Delta p p_{\mathrm{m}}}{\mu v_{0}^{2}}=\frac{\pi a^{4}}{L_{c}} M_{\mathrm{S} 0},
$$

where

$$
M_{\mathrm{S} 0}=\frac{\Delta p p_{m}}{\mu v_{0}^{2}} \quad\left[\frac{\mathrm{kg}}{\mathrm{s}} \mathrm{m}^{-3}\right] .
$$


In the hydrodynamic flow regime the molecule-molecule collisions dominate the molecule-surface collisions. Contrarily, in the free molecular flow regime ( $\delta \leq 0.1$, Knudsen diffusion regime) the molecule-molecule collisions can be practically neglected and the molecule-surface collisions guide the flow. In this regime the mass flow rate is calculated from following expression [27]:

$$
\dot{M}_{\mathrm{FM}}=\frac{2-\alpha}{\alpha} \frac{8}{3 \sqrt{\pi}} \dot{M}_{\mathrm{G}}
$$

with

$$
\dot{M}_{\mathrm{G}}=\frac{\pi a^{3}}{L_{c}} \frac{\Delta p}{v_{0}}=\frac{\pi a^{3}}{L_{c}} M_{\mathrm{G} 0}
$$

where

$$
M_{\mathrm{G} 0}=\frac{\Delta p}{v_{0}} \quad\left[\frac{\mathrm{kg}}{\mathrm{s}} \mathrm{m}^{-2}\right] .
$$

In the case of the transitional flow regime $(0.1 \leq \delta \leq 10)$ the mass flow rate can be found only numerically by the solution of the linearized Boltzmann equation (or of others model kinetic equations [28], [29]). In this flow regime the number of molecule-surface collisions is comparable to the number of intermolecular collisions.

The approximate expression of the dimensionless mass flow rate $G(G=$ $\left.\dot{M} / \dot{M}_{\mathrm{G}}\right)$ through a capillary of the radius $a$ and the length $L_{c}$, which covers all flow regimes, was proposed in [30] for the diffuse scattering, $\alpha=1$ :

$$
G(\delta)=\dot{M} / \dot{M}_{\mathrm{G}}=\frac{8}{3 \sqrt{\pi}} \frac{1+0.04 \delta^{0.7} \ln \delta}{1+0.78 \delta^{0.8}}+\left(\frac{\delta}{4}+\sigma_{p}\right) \frac{\delta}{1+\delta} .
$$

The typical shape of the normalized mass flow rate, $G$ function, Eq. (27), for a capillary is shown on Fig. 3. The function $G$ has the finite limit in the free molecular regime, i.e. when $\delta \rightarrow 0$. In the case of diffuse scattering of the molecules from the surface, $\alpha=1, \lim _{\delta \rightarrow 0} G(\delta)=8 /(3 \sqrt{\pi})$, see Eqs. (24) and (27). In the opposite limit case, $\delta \rightarrow \infty$, the hydrodynamic flow regime, the normalized mass flow rate, $G$ function, Eq. (27), tends to infinity. Therefore, in the following to have a finite value of the mass flow rate in the hydrodynamic flow regime, we will use the representation of the mass flow rate in form (21), which limit case for $\delta \rightarrow \infty$ gives very known Poiseuille flow rate, Eq. (22).

\section{Determination of porous medium characteristics from pressure measurements}

In the previous Section, we introduced the complete description of the flow through a single capillary. In this Section, the model of the porous media as a bundle of capillaries is presented, and its parameters as the capillary radius, capillary number, tortuosity, and specific surface area are extracted from the measurements. The proposed geometrical model corresponds to a homogeneous porous medium with a signature of a single pore size. 


\subsection{Fitting range}

The analytical expressions for the mass flow rate through a single capillary, provided in the previous Section, could be used to calculate the mass flow rate through a microporous sample by representing it as a bundle of capillaries. To choose the analytical expression for the mass flow rate the flow regime in a capillary must be known. However, the flow regimes depend on the rarefaction parameter (Knudsen number), which includes the characteristic dimension of a flow (capillary radius), which is a priori unknown.

To have an idea about the flow regime it is useful to note that the mass flow rate through a capillary in free molecular regime is proportional to $\Delta p / v_{0}$, this motivates our definition of the dimensionless quantity $G_{0}$ as

$$
G_{0}=\dot{M} /\left(\Delta p / v_{0}\right)=\dot{M} / M_{\mathrm{G} 0} \quad\left[\mathrm{~m}^{2}\right] .
$$

By defining the dimensionless quantity $G_{0}$ in this way, we eliminate its dependence from the geometrical parameters, $a$, and $L_{c}$, which are unknown a priori in this model. By analogy, in the hydrodynamic flow regime, the mass flow rate is proportional to $\Delta p p_{m} /\left(\mu v_{0}^{2}\right)$, so we define dimensionless quantity $S_{0}$ as

$$
S_{0}=\dot{M} /\left(\frac{\Delta p p_{m}}{\mu v_{0}^{2}}\right)=\dot{M} / M_{\mathrm{S} 0} \quad\left[\mathrm{~m}^{3}\right] .
$$

When we plot these normalized quantities, $G_{0}$ and $S_{0}$, as a function of inverse molecular mean free path $\ell^{-1}$ and molecular mean free path $\ell$, respectively, we find similar behavior as for the mass flow rate through a tube. That is, with these normalizations we find a constant value in the respective regime and we can make the first identification of two known limits, the free molecular and hydrodynamic regimes.

However, it is more complicated to identify the slip flow regime. The $G_{0}$ curve for the first disc is presented in Fig. 4. It is clear that the analytical curve of dimensionless mass flow rate $G$ trough a single capillary, Fig. 3, has a very similar shape to the experimental curve of the normalized quantity $G_{0}$ through the microporous medium, see Fig. 4. Therefore, by analogy, we can identify the slip flow regimes visually in terms of inverse molecular mean free path. In Table 2 different flow regimes are represented by different molecular mean free path ranges.

When the flow regime is determined the corresponding analytical mass flow rate expression can be chosen and then the measured mass flow rate can be fitted to determine the characteristic flow dimension, pore radius, and the number of capillaries in the representation of the porous medium as a bundle of capillaries. Once the flow dimension has been extracted, it is useful to calculate the Knudsen number and the rarefaction parameter and compare how close the chosen Knudsen number (rarefaction parameter) range is to the classical definition of the slip regime range for a capillary. For the slip flow regime, we should find the Knudsen number $K n \approx 0.1$ and the rarefaction parameter $\delta \approx 10$. We can use this point as a guideline to further refine our definition of the regimes. This is 
done by reiterating the process of fitting and extracting the equivalent flow dimension and again calculating the Knudsen number and rarefaction parameter until become close to the theoretical values of a tube.

Once the definition of the slip flow regime has been done in a suitable way, we have to find three properties which present the additional argumentation that we have defined our regime correctly. First, the Knudsen number and rarefaction parameter are close to the theoretical values for a tube. Second, the relative difference of our linear fit in the slip flow regime and the measured values do not have a trend but is rather scattered around a constant value. Third, the intrinsic permeability is gas independent within experimental uncertainty.

\subsection{Effective pore size}

Let us use the analytical expression for the mass flow rate through one tube, Eqs. (21) and (22), and write it for a bundle of $N$ capillaries, where the capillary length $L_{c}$ can be different from the thickness $L$ of the porous sample (disc). In this case the mass flow rate through a bundle of $N$ capillaries reads:

$$
\dot{M}=\frac{N \pi a^{4}}{L_{c}} \frac{\Delta p p_{m}}{\mu v_{0}^{2}}\left(\frac{1}{4}+\frac{\sigma_{p}}{\delta}\right) .
$$

In the previous expression four parameters are unknown: $N, a, L_{c}$ and $\sigma_{p}$. To determine them from experimental data we can write the previous expression in the following form

$$
S_{0}=\dot{M} / M_{\mathrm{S} 0}=\frac{N \pi a^{4}}{L_{c}}\left(\frac{1}{4}+\frac{\sigma_{p}}{\delta}\right),
$$

where $M_{\mathrm{S} 0}$ is defined in (23). Then we fit previous expression according to the linear regression:

$$
\mathcal{F}_{S}=\mathcal{A}_{S} X+\mathcal{B}_{S},
$$

where

$$
\mathcal{A}_{S}=\sigma_{p} \frac{\pi a^{3} N}{L_{c}}, \quad \mathcal{B}_{S}=\frac{\pi a^{4} N}{4 L_{c}}, \quad X=\ell,
$$

$\mathcal{A}_{S}$ and $\mathcal{B}_{S}$ are the fitting coefficients of the S-fit. The mass flow rate is fitted via the molecular mean free path, $X=\ell$, Eq. (15). From the previous expressions, it is clear that the slope of the fitting curve, $\mathcal{A}_{S}$ coefficient, depends on the gas nature only via the slip coefficient $\sigma_{p}$.

As it was mentioned previously, initially the characteristic dimension $a$ of the flow are not known, and we can refer to different flow regimes only by using the molecular mean free path. The intervals associated with different flow regimes and expressed in term of the molecular mean free path are presented in Table 2. The S-type fit, Eq. (32), is realized in the hydrodynamic and slip flow regimes, so for $\ell>0.19 \mu \mathrm{m}$ for the first disc.

The fitting coefficients, $\mathcal{A}_{S}$ and $\mathcal{B}_{S}$, for the first disc and various gases are shown in Table 3. It is worth to note that even if the measurements for four gases are carried out for the first disc only for two gases, Nitrogen and Argon, 
the number of the experimental points in slip regime is large enough to obtain the good fitting curves. The measured mass flow rate normalized using Eq. (31), and the fitting curve, according to Eq. (32), are shown in Fig. 5 for Argon. The quality of the fit is also tested by plotting the relative deviation between the measured and fitted quantities, $\left(S_{0}-\mathcal{F}_{S}\right) / \mathcal{F}_{S}$, see Fig. 6. As it is clear from this last figure, the points are homogeneously distributed around 0, and they do not show any trend, which confirms the quality of fitting function and supports also our choice of the fitting range, see comments at the end of Section 5.1. The results for other gases are close to that measured for Argon.

From the fitting coefficients, $\mathcal{A}_{S}$ and $\mathcal{B}_{S}$, the effective flow dimension of the porous medium $a$, i.e. effective pore radius, can be found as

$$
a=4 \sigma_{p} \frac{\mathcal{B}_{S}}{\mathcal{A}_{S}} .
$$

To calculate the characteristic dimension of the porous medium, $a$, from the previous expression, Eq. (34), we need only the information on the velocity slip coefficient, $\sigma_{p}$, which characterizes the gas-surface interaction. In addition, Eq. (34) is independent of the external geometrical parameters of a sample, so we are not restricted to only cylindrical shape of the porous media. In the following, we assume that all the gases interact with the wall of the porous medium diffusively and the analytical value of this coefficient $\left(\sigma_{p}=1.018\right)$ is used for further calculations. To estimate the error, induced by this assumption, we calculated the relative (compared to Nitrogen) $\sigma_{p}$ value from following relation

$$
\frac{\sigma_{p}}{\sigma_{p}^{N_{2}}}=\frac{\mathcal{A}_{S} / \mathcal{A}_{S}^{N_{2}}}{\mathcal{B}_{S} / \mathcal{B}_{S}^{N_{2}}} .
$$

This relative value $\sigma_{p} / \sigma_{p}^{N_{2}}$ of the velocity slip coefficient for Argon and the first disc is of the order of

$3 \%$, which is less than the measurement accuracy. Therefore, the assumption on the equality of $\sigma_{p}$ coefficient for analyzed here gases is justified, so we continue to use the same value of the velocity slip coefficient (analytical value 1.018 [26]) for all gases used in the experiments.

To obtain the characteristic flow dimension (effective pore size) we used here the experimental data in the hydrodynamic and slip flow regimes. Another possible approach to calculated the effective pore size from the measurements is presented in AppendixC. This methodology is based on the utilization of the measurements in the free molecular and hydrodynamic flow regimes, but it was not applied in this work.

\section{Other characteristic parameters of porous sample}

As it discussed in previous Section, from the mass flow rate fitting expression we can extract: the characteristic dimension of porous medium, $a$, and also the number $N$ of the capillaries as

$$
N=\frac{\mathcal{B}_{S} L_{c}}{\pi a^{4}} .
$$


However, in Eq. (36) the capillary length, $L_{c}$, is still unknown, so we can make two assumptions to obtain this value. One of possibilities is to assume that the capillary length is equal to the porous disc thickness, $L_{c}=L$, so the tortuosity factor, $l_{\tau}$, Eq. (10), is equal to 1 . However, with this assumption the sample porosity, $\varepsilon=2.2 \%$, is much smaller than that provided by the manufacturer, $15.9 \%$.

The second possible choice is to assume that the capillary length, $L_{c}$, is equal to $l_{\tau} L$. However, to calculate $l_{\tau}$ we have to introduce new additional parameter, the porosity, $\varepsilon$, which can be known either from the manufacturer or from the tomography analysis (see Section 8), then the tortuosity is calculated as

$$
l_{\tau}=\frac{a}{2} \sqrt{\frac{\varepsilon}{\mathcal{B}_{S}}} \frac{S}{L} .
$$

Finally, to have the complete realistic description of a porous sample by using the model of the bundle of the tortuous capillaries we need to use additionally the information about the porosity.

The last important data, which can be extracted from the measurements, also by using additional information about the porosity, is the value of the surface-to-volume ratio, Eq. (13), which can be also calculated as

$$
\mathcal{S}_{\mathrm{A}}=\varepsilon \frac{2}{a} .
$$

These results extracted from the measurements are compared with the results of the computer tomography analysis and with that of the mercury porosimetry in Section 9.

\section{Permeability}

In Section 2.3 we provided the definition of the permeability as it was proposed by Darcy, i.e. for the incompressible fluid, and then its expression through the mass flow rate, Eq. (8), more adapted for the gas flows, so the permeability is calculated as

$$
K=\dot{M} \frac{\mathcal{R} T}{p_{\mathrm{m}}} \frac{\mu}{S} \frac{L}{\Delta p} .
$$

By using the same model of the porous media as a bundle of $N$ capillaries with length $L_{c}$ and replacing the mass flow rate in the previous expression by its representation provided in Section 5.2, Eqs. (30), (31), we expresse the permeability as

$$
K=\dot{M} / M_{\mathrm{K} 0}, \quad M_{\mathrm{K} 0}=\frac{p_{m} \Delta p}{\mu v_{0}^{2}} \frac{2 S}{L}=M_{\mathrm{S} 0} \frac{2 S}{L},
$$

or

$$
K=\frac{\pi a^{4} N}{L_{c}} \frac{L}{2 S}\left(\frac{1}{4}+\frac{\sigma_{p}}{\delta}\right) .
$$


431 The last expression can be rewritten in the form analogous to Eq. (32) and then

432 fitted according to the linear regression:

$$
\mathcal{F}_{K}=\mathcal{A}_{K} X+\mathcal{B}_{K}
$$

433 where

$$
\mathcal{A}_{K}=\sigma_{p} \frac{\pi a^{3} N}{L_{c}} \frac{L}{2 S}, \quad \mathcal{B}_{K}=\frac{\pi a^{4} N}{4 L_{c}} \frac{L}{2 S}, \quad X=\ell,
$$

${ }_{434} \mathcal{A}_{K}$ and $\mathcal{B}_{K}$ are the fitting coefficients. By comparing the coefficients $\mathcal{A}_{K}$ and ${ }_{435} \mathcal{B}_{K}$ of the permeability fit, Eq. (43), and coefficients $\mathcal{A}_{S}$ and $\mathcal{B}_{S}$, Eq. (33), for 436 the fit of the mass flow rate, Eq. (32), one can see that they differ only in the ${ }_{437}$ factor $L /(2 S)$ and the pore characteristic dimension can be also found from the ${ }_{438}$ ratio of coefficients $\mathcal{A}_{K}$ and $\mathcal{B}_{K}$, as it was done in Section 5.2 for $\mathcal{A}_{S}$ and $\mathcal{B}_{S}$ 439 coefficients.

$440 \quad$ From Eq. (41) we also obtain the well known Klinkenberg expression

$$
K=K_{\mathrm{D}}\left(1+4 \frac{\sigma_{p}}{\delta}\right)=K_{\mathrm{D}}\left(1+4 \sigma_{p} K n\right),
$$

${ }_{441}$ where $K_{\mathrm{D}}$ is the hydrodynamic (Darcy or intrinsic) permeability

$$
K_{\mathrm{D}}=\frac{\pi a^{4}}{8} \frac{N L}{L_{c} S}=\frac{\varepsilon}{l_{\tau}^{2}} \frac{a^{2}}{8},
$$

442 which can be found from the fitting coefficient $\mathcal{B}_{K}$ (or $\mathcal{B}_{S}$, obtained from the fit 443 of the mass flow rate) as

$$
K_{\mathrm{D}}=\mathcal{B}_{K}=\mathcal{B}_{S} \frac{L}{2 S} .
$$

444 As it is clear from Eq. (44), in the slip flow regime, the permeability becomes ${ }_{445}$ inversely proportional to the rarefaction parameter, i.e. to the mean pressure. ${ }_{446}$ Therefore, we can rewrite Eq. (44) in the following form, proposed initially by 447 Klinkenberg [16],

$$
K=K_{\mathrm{D}}\left(1+\frac{b}{p_{m}}\right),
$$

448 here $b$ is a gas dependent coefficient. By identifying Eqs. (44) and (47) we can 449 deduce the expression for $b$ :

$$
b=4 \sigma_{p} \frac{\mu v_{0}}{a} \quad[\mathrm{~Pa}] .
$$

450 It is clear that $b$ coefficient depends not only from the gas nature through the gas 451 viscosity, the most probable molecular velocity and the velocity slip coefficient, ${ }_{452}$ but also from the characteristic dimension $a$ (effective pore radius) of the porous 453 medium, therefore, expression (47) is not at all universal.

${ }_{454}$ It is worth to note that the Klinkenberg expression, Eq. (47), is derived 455 from the expression of the mass flow rate through a bundle of capillaries in ${ }_{456}$ the slip flow regime, so, theoretically, expression (47) is valid only in the slip 
flow regime. To find the limits of the validity of the Klinkenberg expression the model proposed in [30] for the mass flow rate through a single capillary for all flow regimes, Eq. (27), can be used:

$$
K=G \frac{K_{\mathrm{D}}}{4 \delta} .
$$

The limits of the validity of the Klinkenberg expression for the analyzed porous samples are discussed in Section 9.

\section{Tomography analysis}

To have additional information about the samples, a typical sample from the same batch was scanned with MicroXCT-400 tomograph at CEREGE, ${ }^{1}$ which uses the linear attenuation method. The focal spot size of X-ray beam was 5-7 $\mu \mathrm{m}$. The geometrical voxel size is determined by the size and number of detector elements and the source-object-detector distances (magnification). The microXCT-400 is also equipped with many optical lenses that lead to an additional optical magnification. In this work, an x40 optical lens was used [31]. Finally, the geometrical voxel size is fixed to $1.8 \mu \mathrm{m}$. The image stack corresponds to a cylinder of $1.8 \mathrm{~mm}$ of diameter and $1.8 \mathrm{~mm}$ thick composed of 1000 slices of $1000 \times 1000$ voxels size. The porous morphological analysis was perform with the iMorph software [31], [9] on a cubic Region Of Interest (ROI) made of $500 \times 500 \times 500$ voxels, see Fig. 7 , which represents $0.35 \%$ of the total volume of the analyzed sample.

The pore network segmentation is a crucial step consisting to binarize the reconstructed volume from X-ray computed tomography acquisitions. Because our 3D images of porous sample are under resolved and weakly contrasted, hysteresis method is well adapted for the binarization compared to classical Otsu binarization method which results in our case to a mixing of phases (i.e. the solid and poral phases). The hysteresis function [32] performs a dual thresholding operation on the original grayscale image using two threshold values (lower and upper). For the specific application of membrane binarization, the lower threshold is chosen as the smaller threshold that allows the percolation of the poral network and the upper threshold is tuned to obtain the porosity given by the manufacturer [33].

The local thickness is computed for every voxel of the poral space by filling the pore space with spheres

[34], [35]. The volumetric distribution of the local thickness gives the mean pore size diameter equal to $7.26 \pm 5.31 \mu \mathrm{m}$. Its cumulative distribution (Fig. 8) shows that $65 \%$ of the total pore volume is composed of structures with local thickness inferior to $5 \mu \mathrm{m}$, and that $80 \%$ of the total pore volume is occupied by structures with local thickness inferior to $7 \mu \mathrm{m}$.

${ }^{1}$ Centre for Research and Teaching in Environmental Geoscience, Aix Marseille University, Aix-en-Provence, France, https://www.cerege.fr 
To quantify the size of the constrictions in the vicinity of the pores, we use a watershed pore segmentation method based on the ultimates eroded. The watershed operator [36] that is used to individualize each pore surrounded by constrictions, relies on iterative erosion and dilation. The Euclidean distance of the pixel to the nearest background pixel is therefore called the Euclidean distance map. The erosion process (that consist in peeling the distance map successively) needs to be constrained such that the isolated pixels (i.e. pixels surrounded by eight neighbors in each and any erosion iteration) may not be eroded. These pixels coincide with the local maxima in the Euclidean distance map and are called ultimate eroded points [37], because further constrains erosions do not change the image of ultimate's points. The ultimate eroded points are now iteratively dilated through the watershed region growing process and following the distance map values (Fig. 9). The voxels that correspond to the meeting region coming from different ultimate's points labels are identified as throats. For every throat surface we compute the equivalent ellipsoid and we report here the distribution values of the major and the minor axis (Fig. 9). The minor axis length gives the diameter of the maximal inscribed disk into the throat. The mean values are $7.7 \mu \mathrm{m}$ and $18.8 \mu \mathrm{m}$ for the minor and major axis, respectively.

We compute the penetration length of the porous sample for different particle diameters [38]. The results are presented on Figure 10. From this information we can the easily deduce the geometrical cutt-off size of the membrane. From Figure 10, it is clear that only the particles with a diameter of around $3.5 \mu \mathrm{m}$ are able to cross the porous sample.

\section{Results and comparison}

In this Section we present the results obtained with the proposed methodology on the effective pore size, tortuosity, surface-to-volume ratio and the permeability. We compare these porous sample characteristics to the data obtained from the tomographic and porosimetry analyses, when they are available.

\subsection{Pore size}

We start by the analysis of the pore size with one porous sample from a batch, called previously first disc. Applying the presented here experimental methodology, explained in details in Section 5.2, the effective pore dimensions are extracted from the mass flow rate measurements,

see Table 4. By analyzing the data presented in Table 4, we can see that for this porous sample the effective pore diameters, $2 a$, calculated with different gases, are very close one to another. The uncertainty in the estimation of the characteristic pore dimension is of the order of $16 \%$ for Nitrogen and decreases up to $13.9 \%$ for Argon. The average pore dimension, estimated with two gases, $2 a=3.6 \mu \mathrm{m}$, is obtained with an uncertainty of $13.9 \%$.

The uncertainty of the effective pore dimension is calculated using the square root of the summation of the fitting coefficients uncertainty, which is calculated from the limits of a $95 \%$ confidence interval of the fitting parameters. 
The iMorph computer analysis of the tomographic data, see Section 8, allows to obtain the representation of the porous structure of a sample as the system of the pores which are connected by the constrictions (throats). From the analysis of the aperture map distribution, Fig. 8, it was found that $65 \%$ of total pore volume is composed of the structures with the local thickness smaller that $5 \mu \mathrm{m}$, which is close to the results found for the first disc. From the distribution of the throat size dimensions, shown on Fig. 9, it is clear that $25 \%$ of the throats have the dimension equal to $3.6 \mu \mathrm{m}$. In addition, from the iMorph analysis it was also found that only the particles with diameter of around $3.5 \mu \mathrm{m}$ are able to cross the sample, see Fig. 10.

All this information confirms our experimental finding for the first disc. From this analysis, we can conclude that the proposed gas flow methodology allows us to estimate the effective pore size which determines the flow through a porous medium. This dimension is also correlated to the throat size dimension and to the particle cut-off dimension, obtained from the tomography analysis.

\subsection{Tortuosity and surface-to-volume ratio}

The information about the effective pore dimension is extracted without any additional knowledge about the analyzed porous sample. However, if we assume that the sample porosity is equal to $13.6 \%$, the value obtained from the tomographic analysis, then additional characteristics of the porous sample are obtained: the tortuosity, Eq. (37), the capillaries number, $N$, Eq. (36), and surface-to-volume ratio, $\mathcal{S}_{\mathrm{A}}$, Eq. (38). All these values are presented in Table 4 for the first disc. These characteristics can be compared with the data on the tortuosity and surface-to-volume ratio, obtained from the tomography analysis, see Table 5. The tortuosity in three directions is given in Table 5 and the gas permeation direction corresponds to the $z$ axis. The computer tomography tortuosity in this direction is equal to 1.61 , which is close to the tortuosity calculated for the first disc, 2.5, see Table 4.

The averaged over two gases value of the surface-to-volume ratio is equal to $15.0 \times 10^{4} \mathrm{~m}^{2} / \mathrm{m}^{3}$ for the first disc, see Table 4 . This value is of the same order of magnitude as that provided by the tomographic analysis, $6.27 \times 10^{4}$ $\mathrm{m}^{2} / \mathrm{m}^{3}$, see Table 5 .

Both data on the tortuosity and surface-to-volume ratio, found from the proposed methodology, are close to that obtained from the tomography analysis.

\subsection{Non-destructive analysis}

To check the repeatability of the determination of the effective pore size dimension, we have analyzed the second disc, which was provided by the same manufacturer, so supposed to be identical to the first disc.

The same analysis was carried out for this second disc and the effective pore diameters, $2 a$, calculated with three gases are provided in Table 4 . As for the first disc, the effective pore diameters obtained with different gases are very close one to another. The uncertainty in the estimation of the characteristic pore dimension is of the order of $16 \%$ for Argon and decreases up to $12 \%$ and $10 \%$ 
for Helium and Nitrogen, respectively. The average pore dimension, estimated with three gases, $2 a=22 \mu \mathrm{m}$, is obtained with an uncertainty of $13.6 \%$. The much larger effective pore size, $22 \mu \mathrm{m}$, found for the second disc, represents a possible sample imperfection: the largest pores are interconnected for this sample and so they determine the gas flow rate. This structural defect is also visible through the mercure porosimetry analysis of a sample from the second batch and it results in a peak between 20 and 30 microns, see Fig. 11.

This finding suggests that the proposed gas flow method could potentially be used as a method of the non-destructive analysis of a porous sample.

\subsection{Permeability}

The hydrodynamic (intrinsic) permeability $K_{D}$, calculated from the mass flow rate measurements, is provided in Table 6, for the first and second discs, respectively. As it is clear from Table 6 the hydrodynamic permeability is gas independent within the experimental uncertainty and it is found to be much smaller for the second disc. It is also worth to underline that two parameters for description of the permeability in form of Eq. (44), $a$ and $K_{\mathrm{D}}$, are obtained directly from the fit of the measured mass flow rate or permeability data without any additional information about the sample porosity.

The intrinsic permeability is also provided by iMorph computer analysis, based on the analytical relation for the channel conductivity, and it is equal to $1.3 \times 10^{-14} \mathrm{~m}^{2}$. This value is close to that obtained for the first disc, $0.9 \times 10^{-14}$ $\mathrm{m}^{2}$, see Table 6 .

The permeability for both discs is plotted on Fig. 12 as a function of the Knudsen number. The permeability increases in more than two orders of magnitude (first disc) when the mean pressure is decreasing.

In Table 6 the $b$ coefficient is provided for two porous discs and it is two times smaller for the first disc compared to the second one which confirms that the Klinkenberg expression is not universal and the $b$ coefficient is gas and porous sample dependent.

The measured and calculated from Eqs. (49), (27) permeabilities are shown on Fig. 13. Very good agreement between experimental and analytical data are found in the near hydrodynamic, slip and beginning of the transitional flow regimes, see Fig. 13. However, in the free molecular flow regime, the semianalytical expression overestimate the experimental data. It can be explained by the fact that the experimental data are fitted only in the slip flow regime. Therefore, the deviation between measured and semi-analytical data is found for the large Knudsen number range. It also confirms that the Klinkenberg expression is valid in the slip regime only.

The implementation of Eqs. (49), (27) shows an interesting potential of extracting intrinsic permeability from apparent permeability in free molecular and transitional regimes. 


\section{Conclusion}

The classical model of the porous media presentation as a bundle of capillaries was revised. The original methodology was suggested to determine the characteristic flow dimension. The experimental procedure is developed to determine the effective pore size (characteristic flow dimension) and the number of capillaries, related to the model a bundle of capillaries. The experimentally obtained effective pore dimension is in very good agreement with the results of the mercury porosimetry and micro-computed tomography. The use of additional information on the sample porosity allows to find the tortuosity and the surface-to-volume ratio, which were close to that calculated from the tomography analysis. The Klinkenberg formula was also analyzed, and it was shown that this expression is not general and $b$ coefficient reveals gas and porous medium dependency. In addition, the Klinkenberg expression is valid only for the slip flow regime, which was shown experimentally. Therefore, this formula has to be used with precaution in the case of low porous structures. The intrinsic permeability obtained by tomography analysis is very close to the measured permeability, which is not surprising in the case of the homogeneous porous medium. The proposed approach is the first very promising stage to evolve towards measurements of even lower permeabilities and also the characteristic dimension (pore size) of membranes used for microfiltration (>100 nm ) and ultrafiltration $(>10 \mathrm{~nm})$.

\section{Acknowledgement}

The project leading to this publication has received funding from Excellence Initiative of Aix-Marseille University - A*MIDEX, a French "Investissements d'Avenir" programme. It has been carried out in the framework of the Labex MEC. The authors (M.V. Johansson, P. Perrier, and I. Graur) would like to acknowledge the financial support provided by the European Union network program H2020, MIGRATE project under Grant Agreement No.643095.

[1] S. P. Adiga, C. Jin, L. A. Curtis, N. A. Monteiro-Riviere, R. J. Narayan, Nanoporous membranes for medical and biological applications, WIREs Nanomedicine and Nanobiotechnology Advances Reviews 1 (568-581).

[2] R. Abedini, A. Nezhadmoghadam, Application of membrane in gas separation processes: its suitability and mechanisms, Petroleum and Coal 52 (2010) 69 .

[3] Y. S. Lin, A. J. Burggraaf, Experimental studies on pore size change of porous ceramic membranes after modification, Journal of Membrane Science 79 (1993) 65-82.

[4] M. E. Naraghi, F. Javadpour, A stochastic permeability model for the shalegas system, International Journal of Goal Geology 14 (2015) 111-124. 
[5] S. Lowell, J. E. Shield, M. A. Thomas, M. Thommes, Characterization of Porous Solids and Powders: Surface Area, Pore Size and Density (Particle Technology Series), 4th Edition, Springer, 2006.

[6] S. Brunauer, P. H. Emmett, E. Teller, Adsorption of gases in multilayers, J. Am. Chem. Soc 60 (1938) 309-319.

[7] Y. Wyart, G. Georges, L. Arnaud, C. Deumie, C. Amra, P. Moulin, Membrane characterisation by optical methods: Ellipsometry of the scattered field, J. Membrane Sci. 318 (2008) 145-153.

[8] Y. Wyart, G. Georges, C. Deumie, C. Amra, P. Moulin, Membrane characterization by microscopic methods: multiscale structure, J. Membrane Sci. 315 (2008) 82-92.

[9] C. El Hachem, K. Abahri, J. Vicente, R. Bennacer, R. Belarbi, Hygromorphic characterization of softwood under high resolution x-ray tomography for hygrothermal simulation, Heat and Mass Transfer (3) (2018) 1-9.

[10] M. Rojas Cardenas, I. Graur, P. Perrier, J. G. Méolans, Thermal transpiration flow: a circular cross-section microtube submitted to a temperature gradient, Phys. Fluids 23 (2011) 031702.

[11] M. V. Johansson, F. Testa, I. Zaier, P. Perrier, J. P. Bonnet, P. Moulin, I. Graur, Mass flow rate and permeability measurements in microporous media, Vacuum 158 (2018) 75-85.

[12] J. Kozeny, Uber kapillare leitung der wasser in boden, Royal Academy of Science, Vienna, Proc. Class I 136 (1927) 271-306.

[13] P. C. Carman, Fluid flow through granular beds, Trans. Inst. Chem. Eng. 15 (1937) 150-166.

URL https://ci.nii.ac.jp/naid/10003392892/en/

[14] J. Cai, E. Perfect, C.-L. Cheng, X. Hu, Generalized modeling of spontaneous imbibition based on Hagen-Poiseuille flow in tortuous capillaries with variably shaped apertures, Langmuir 30 (2014) 5142-5151.

[15] A. Golparvar, Y. K. Yingfang Zhou, Y Kejian Wu, J. Ma, Z. Yu, A comprehensive review of pore scale modeling methodologies for multiphase flow in porous media, advanced in Geo-Energy Research 2 (4) (2018) 418-440.

[16] L. J. Klinkenberg, The permeability of porous media to liquid and gases, Drilling and Production Practice, Amarican Petroleum Institute (1941) 200-213.

[17] T. Ewart, P. Perrier, I. A. Graur, J. G. Méolans, Mass flow rate measurements in gas micro flows, Experiments in Fluids 41 (3) (2006) 487-498. 
[18] M. Rojas-Cardenas, I. Graur, P. Perrier, J. G. Méolans, Time-dependent experimental analysis of a thermal transpiration rarefied gas flow, Phys. Fluids 25 (2013) 072001.

[19] W. F. Brace, J. B. Walsh, W. T. Frangos, Permeability of granite under high pressure, Journal of Geophysical Research 73 (6) (1968) 2225-2236.

[20] D. D. Do, Adsobtion analysis: equilibria and kinetics, Vol. 2, Imperial College Press, Imperial College London SW7 2BT, 1998.

[21] G. A. Bird, Molecular Gas Dynamics and the Direct Simulation of Gas Flows, Oxford Science Publications, Oxford University Press Inc., New York, 1994.

[22] N. Epstein, On tortuosity and tortuosity factor in flow and diffusion through porouse media, Chem. Eng. Sci. 44 (3) (1989) 777-779.

[23] C. Cercignani, Theory and application of the Boltzmann equation, Scottish Academic Press, Edinburgh, 1975.

[24] C. Cercignani, Mathematical methods in kinetic theory, Premuim Press, New York, London, 1990.

[25] S. K. Loyalka, N. Petrellis, S. T. Stvorick, Some numerical results for the bgk model: thermal creep and viscous slip problems with arbitrary accommodation of the surface, Physics of Fluids 18 (1975) 1094.

[26] F. Sharipov, Data on the velocity slip and temperature jump on a gas-solid interface, J.Phys. Chem. Ref. Data 40 (2) (2011) 023101-1-28.

[27] F. Sharipov, Rarefied gas dynamics. Fundamentals for research and practice, WILEY-VCH Verlag GmBH \& Co. KGaA. Weinheim, 2016.

[28] F. Sharipov, V. Seleznev, Data on internal rarefied gas flows, J. Phys. Chem. Ref. Data 27 (3) (1998) 657-706.

[29] I. A. Graur, F. Sharipov, Gas flow through an elliptical tube over the whole range of the gas rarefaction, European Journal of Mechanics/B Fluids 27 (3) (2007) 335-345.

[30] F. Sharipov, I. Graur, C. Day, Leak rate of water into vacuum through microtubes, Journal of Vac. Sci. Technol. A 28 (3) (2010) 443-448.

[31] J. Vicente, Y. Wyart, P. Moulin, Characterization (2d-3d) of ceramic microfiltration membrane by synchrotron radiation: new and abraded membranes, Journal of Porous Media 16 (2013) 537-545.

[32] J. Canny, A computational approach to edge detection, IEEE Transactions on pattern analysis and machine intelligence PAMI-8 (6) (1986) 679-698. 
[33] J. Perrin, J. Vicente, J. P. Bonnet, D. Borscheck, C. Savaro, J. Anquetil, P. Moulin, Morphological characterization of ceramic membranes from $3 \mathrm{~d} x-$ ray computed tomography, in: Multi-scale Materials Under the Nanoscope, 2016 .

[34] J. Remigy, M. Meireles, X. Thibault, Morphological characterization of a polymeric microfiltration membrane by synchrotron radiation computed microtomography, Journal of Membrane Science 305. doi:10.1016/j. memsci.2007.06.059.

[35] N. Bossa, P. Chaurand, J. Vicente, D. Borschneck, C. Levard, O. AguerreChariol, J. Rose, Micro- and nano-x-ray computed-tomography: A step forward in the characterization of the pore network of a leached cement paste, Cement and Concrete Research 67 (2015) 138 - 147. doi:10.1016/ j.cemconres.2014.08.007.

[36] H. Digabel, C. Lantuejoul, Iterative algorithms, Proceedings of the 2nd European Symposium Quantitative Analysis of Microstructures in Material Science, Biology and Medicine (1978) 85-89.

[37] C. Lantuejoul, S. Beucher, On the use of the geodesic metric in image analysis, Journal of Microscopy 121 (1) (1981) 39-49. arXiv:https: //onlinelibrary.wiley.com/doi/pdf/10.1111/j.1365-2818.1981. tb01197.x, doi:10.1111/j.1365-2818.1981.tb01197.x. URL https://onlinelibrary.wiley.com/doi/abs/10.1111/j. 1365-2818.1981.tb01197.x

[38] J. Perrin, J. Vicente, J. P. Bonnet, D. Borscheck, J. Anquetil, P. Moulin, Determination of molecular weight cut off by x-ray micro ct, in: Euromembrane, 2018.

[39] R. Reid, J. Prausnitz, T. Sherwood, The properties of gases and liquids, fourth edition Edition, no. 30 in McGraw-Hill chemical engineering series, McGraw-Hill, 1987.

URL https://books .google.fr/books?id=ZnAfAAAAMAAJ

[40] P. Z. S. Spakovszky, Thermodynamics and propulsion (September 2017).

URL http://web.mit.edu/16. unified/www/FALL/thermodynamics/ notes/node11.html 


\begin{tabular}{|l|c|c|}
\cline { 2 - 3 } \multicolumn{1}{c|}{} & 1st disc & 2nd disc \\
\hline Regime & $\ell[\mu \mathrm{m}]$ & $\ell[\mu \mathrm{m}]$ \\
\hline \hline HYDRO & $1.9 \times 10^{-3}<\ell$ & $0.11<\ell$ \\
\hline SLIP & $0.19<\ell$ & $1.1<\ell$ \\
\hline FM & $19>\ell$ & $110>\ell$ \\
\hline
\end{tabular}

Table 2: Flow regimes identification.

\begin{tabular}{|c|c|c|c|c|}
\cline { 2 - 5 } \multicolumn{1}{c|}{} & \multicolumn{2}{c|}{ 1st disc } & \multicolumn{2}{c|}{ 2nd disc } \\
\hline GAS & $A_{S}\left[10^{-9} \mathrm{~m}^{2}\right]$ & $B_{S}\left[10^{-16} \mathrm{~m}^{3}\right]$ & $A_{S}\left[10^{-9} \mathrm{~m}^{2}\right]$ & $B_{S}\left[10^{-15} \mathrm{~m}^{3}\right]$ \\
\hline \hline $\mathrm{He}$ & & & $2.4 \pm 0.2$ & $7.1 \pm 0.1$ \\
\hline $\mathrm{N}_{2}$ & $1.2 \pm 0.2$ & $5.5 \pm 0.1$ & $2.9 \pm 0.2$ & $7.2 \pm 0.1$ \\
\hline $\mathrm{Ar}$ & $1.2 \pm 0.1$ & $5.5 \pm 0.1$ & $2.4 \pm 0.4$ & $7.4 \pm 0.2$ \\
\hline $\mathrm{AVR}$ & $1.2 \pm 0.1$ & $5.5 \pm 0.1$ & $2.6 \pm 0.3$ & $7.2 \pm 0.1$ \\
\hline
\end{tabular}

Table 3: Fitting coefficients $\mathcal{A}_{S}$ and $\mathcal{B}_{S}$ with $S$-fit.

763

764

765

766
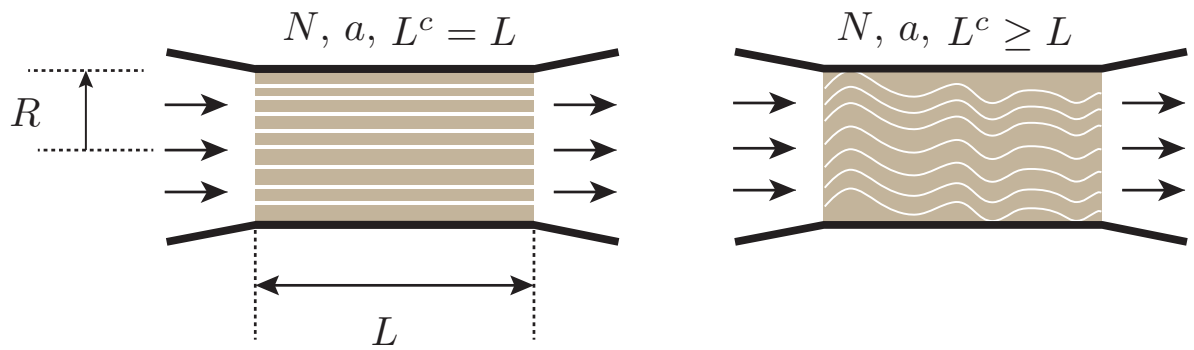

Figure 1: Models of the flat ceramic microporous membrane: bundle of $N$ parallel capillaries of the same radius $a$ (left) of the same length $L_{c}$ equal to the membrane thickness $L$; (right) of the capillary length $L_{c}$ is greater than the membrane thickness $L, L_{c}=l_{\tau} L$. 
1 st disc

\begin{tabular}{|c|c|c|c|c|}
\hline GAS & $2 a[\mu \mathrm{m}]$ & $N\left[10^{5}\right]$ & $\mathcal{S}_{\mathrm{A}}\left[10^{5} \mathrm{~m}^{2} / \mathrm{m}^{3}\right]$ & $l_{\tau}$ \\
\hline \hline $\mathrm{N}_{2}$ & $3.7 \pm 0.6$ & $3.4 \pm 1.0$ & $1.5 \pm 0.6$ & $2.6 \pm 0.4$ \\
\hline $\mathrm{Ar}$ & $3.6 \pm 0.5$ & $3.8 \pm 1.0$ & $1.5 \pm 0.6$ & $2.5 \pm 0.4$ \\
\hline $\mathrm{AVR}$ & $3.6 \pm 0.5$ & $3.8 \pm 0.9$ & $1.5 \pm 0.6$ & $2.5 \pm 0.4$ \\
\hline \multicolumn{5}{|c|}{2 2nd disc } \\
\hline $\mathrm{GAS}$ & $2 a[\mu \mathrm{m}]$ & $N$ & $\mathcal{S}_{\mathrm{A}}\left[10^{4} \mathrm{~m}^{2} / \mathrm{m}^{3}\right]$ & $l_{\tau}$ \\
\hline \hline $\mathrm{He}$ & $25 \pm 3$ & $4300 \pm 900$ & $2.2 \pm 0.7$ & $4.7 \pm 0.6$ \\
\hline $\mathrm{N}_{2}$ & $20 \pm 2$ & $8000 \pm 1000$ & $2.7 \pm 0.6$ & $3.8 \pm 0.4$ \\
\hline $\mathrm{Ar}$ & $25 \pm 4$ & $4000 \pm 1000$ & $2.0 \pm 1$ & $4.7 \pm 0.9$ \\
\hline $\mathrm{AVR}$ & $22 \pm 3$ & $6000 \pm 1000$ & $2.4 \pm 0.9$ & $4.3 \pm 0.6$ \\
\hline
\end{tabular}

Table 4: Estimation of the porous media characteristic dimension, $a$, the number of capillaries $N$, and the surface to volume ratio, $\mathcal{S}_{\mathrm{A}}$, by using S-type fit and the porosity obtained from the micro-computed tomography, $\varepsilon=13.6 \%$. The tortuosity is calculated from Eq. (37).

\begin{tabular}{|c|c|c|c|c|}
\cline { 3 - 5 } \multicolumn{2}{c|}{} & \multicolumn{3}{c|}{$\ell_{\tau}$} \\
\hline$\varepsilon$ & $\mathcal{S} \times 10^{4}\left[\mathrm{~m}^{2} / \mathrm{m}^{3}\right]$ & $x$ & $y$ & $z$ \\
\hline $13.6 \%$ & 6.27 & $2.7 \pm 1.5$ & $2.8 \pm 1.6$ & $1.6 \pm 0.5$ \\
\hline
\end{tabular}

Table 5: Results from iMorph analysis of the one part of the first porous disc, obtained with $1.8 \mu \mathrm{m}$ space resolution: porosity, $\varepsilon$, specific surface area, $\mathcal{S}_{\mathrm{A}}$, and tortuosity, $l_{\tau}$, in three directions $(x, y$ and $z)$.

\begin{tabular}{|c|c|c|c|c|}
\cline { 2 - 5 } \multicolumn{1}{c|}{} & \multicolumn{2}{c|}{ 1st disc } & \multicolumn{2}{c|}{ 2nd disc } \\
\hline GAS & $K_{\mathrm{D}}\left[10^{-15} \mathrm{~m}^{2}\right]$ & $b[\mathrm{kPa}]$ & $K_{\mathrm{D}}\left[10^{-13} \mathrm{~m}^{2}\right]$ & $b[\mathrm{kPa}]$ \\
\hline \hline $\mathrm{He}$ & & & $1.16 \pm 0.02$ & $7.3 \pm 0.8$ \\
\hline $\mathrm{N}_{2}$ & $8.9 \pm 0.2$ & $17 \pm 2$ & $1.16 \pm 0.02$ & $3 \pm 0.2$ \\
\hline $\mathrm{Ar}$ & $9.0 \pm 0.2$ & $18 \pm 3$ & $1.20 \pm 0.03$ & $2.6 \pm 0.5$ \\
\hline $\mathrm{AVR}$ & $9.0 \pm 0.2$ & $18 \pm 3$ & $1.18 \pm 0.02$ & $4.3 \pm 0.5$ \\
\hline
\end{tabular}

Table 6: Klinkenberg coefficients. 

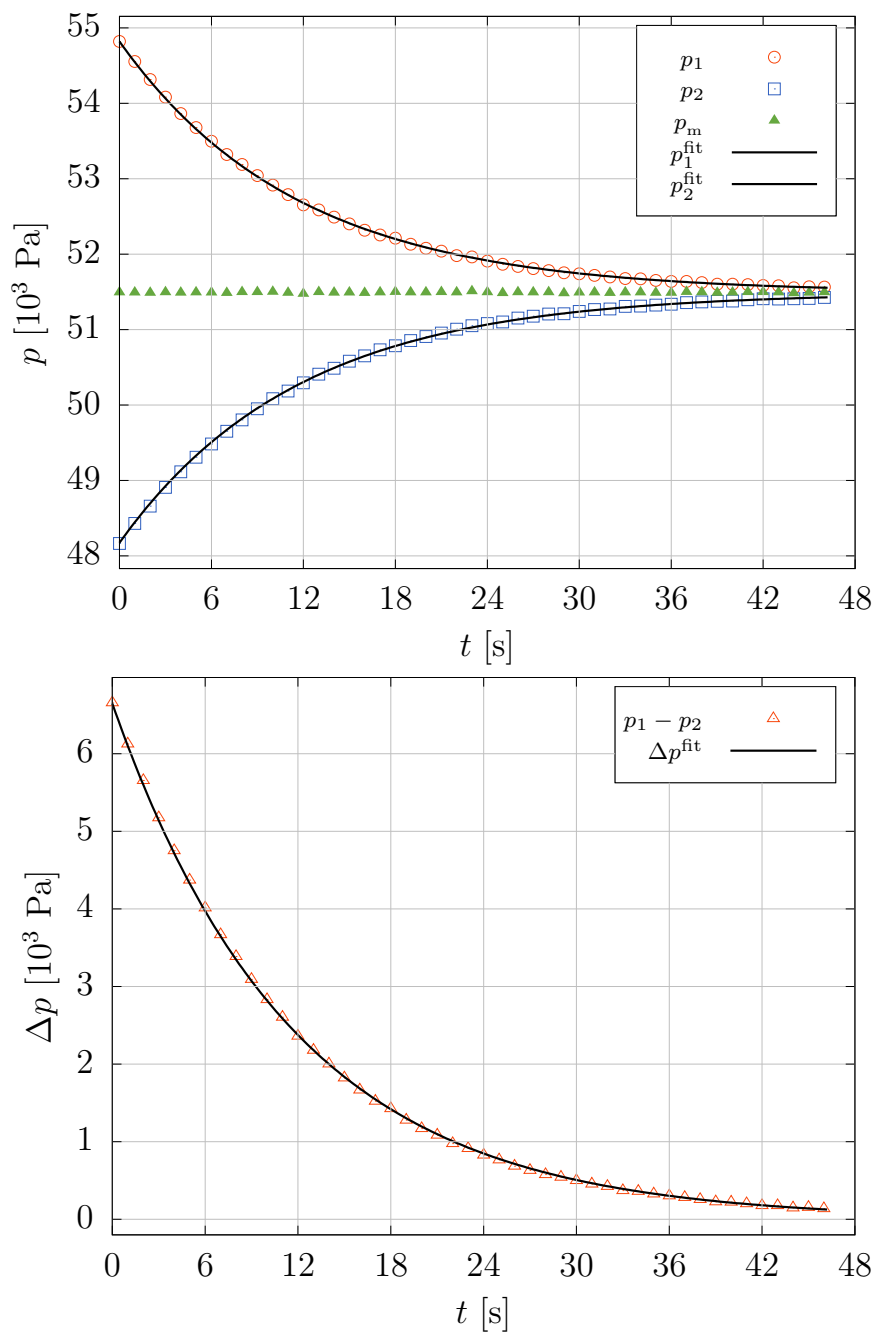

Figure 2: Upper figure: Pressure evolution in time, where the upstream tank pressure, $p_{1}$, is red and the downstream tank pressure, $p_{2}$, is blue, together with the respective fitting curves of the pressure evolution, in black, and the mean pressure $p_{\mathrm{m}}$ in grey. Lower figure shows the evolution of the pressure difference and the exponential fitting of the measurements. 


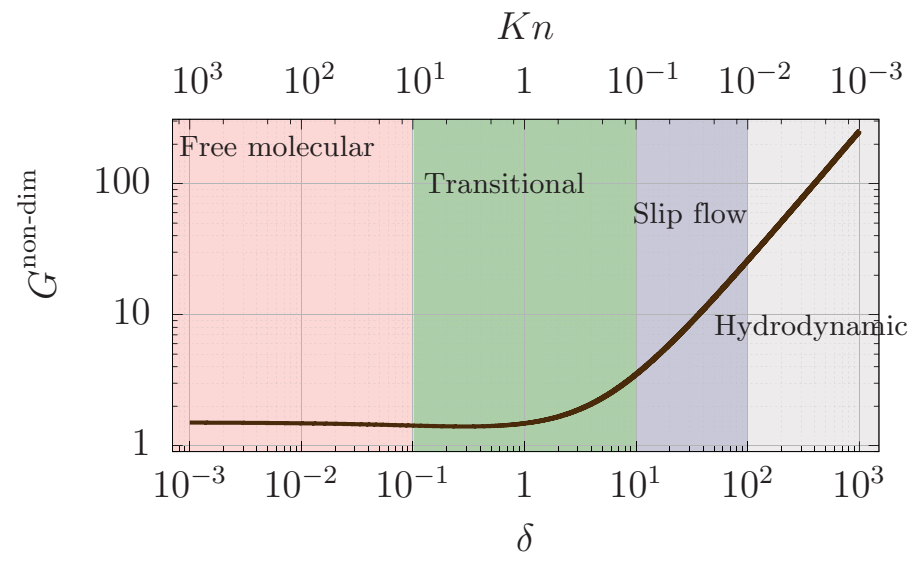

Figure 3: Dimensionless mass flow rate $G$ for a single circular tube, Eq.(27), as a function of rarefaction parameter and Knudsen number.

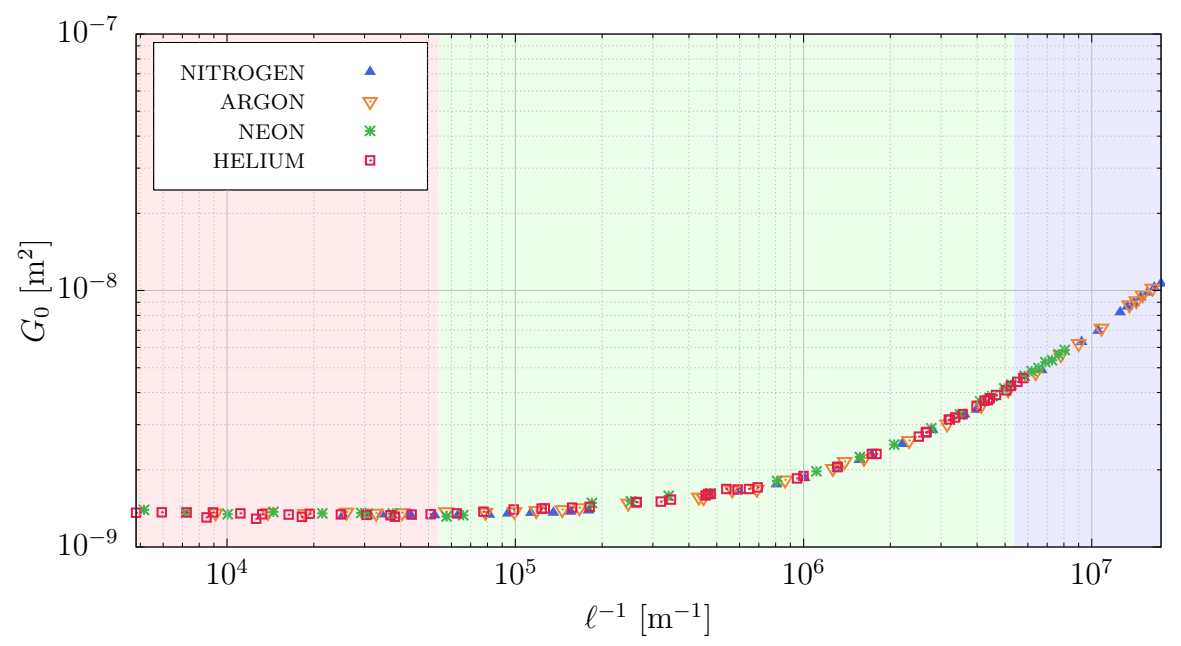

Figure 4: Experimental points for the first disc in normalized form of $G_{0}$ function, $G_{0}=$ $\dot{M} /\left(\Delta p / v_{0}\right)$ as a function of the inverse molecular mean free path, $\ell^{-1}$. 


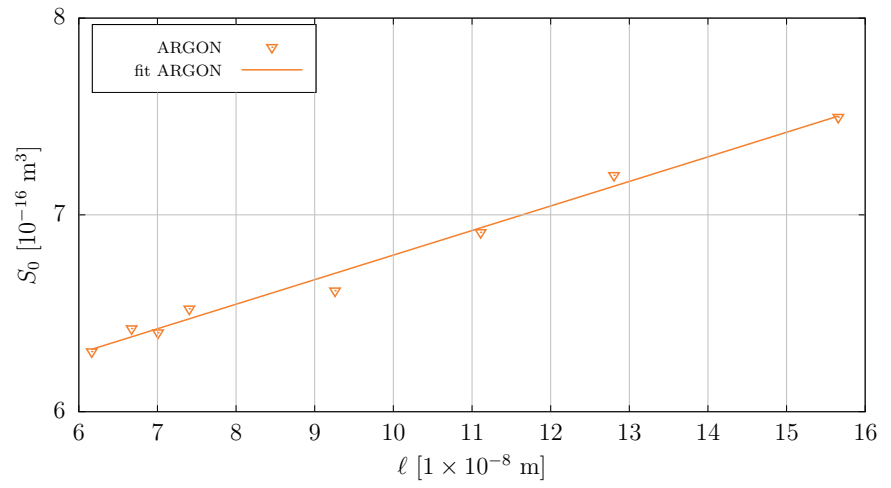

Figure 5: Experimental points of normalized mass flow rate, $S_{0}$, Eq. (31), and the corresponding fitting function, Eq. (32), as a function of the mean free path, $\ell$.

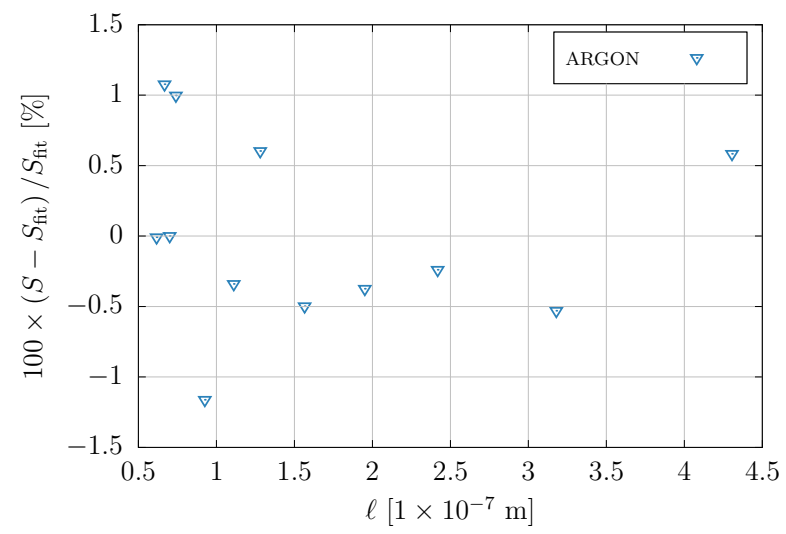

Figure 6: Difference between the experimental points for normalized mass flow rate, Eq. (31), and the fitting curve, Eq. (32), in form $\left(S_{0}-\mathcal{F}_{S}\right) / \mathcal{F}_{S}$ as a function of molecular mean free path, $\ell$. 


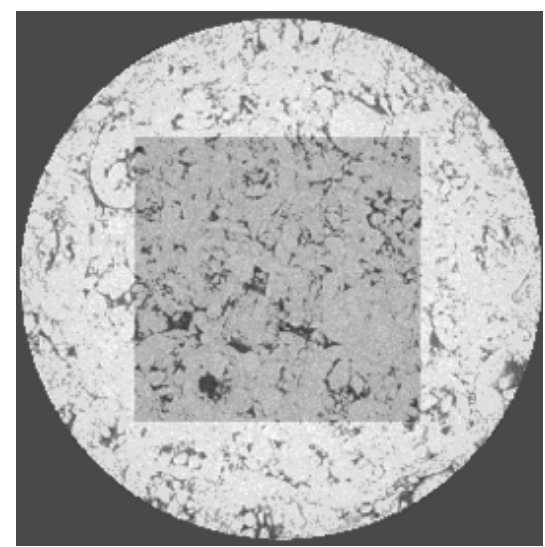

(a)

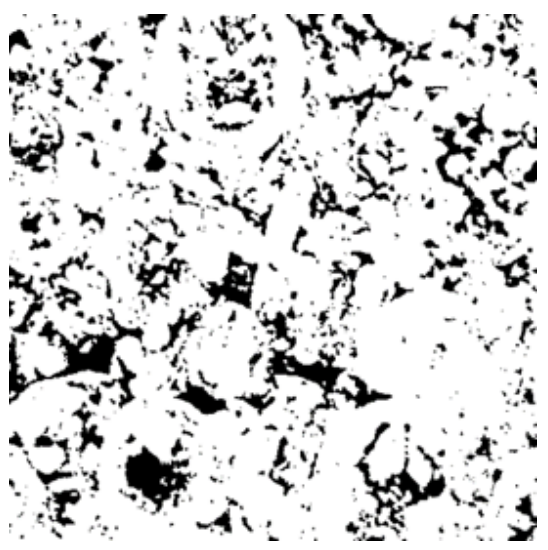

(b)

Figure 7: (a) X-ray reconstructed slice (1000x1000 voxels) and centered cubic Region Of Interest $(500 \times 500$ voxels) use for the analysis (voxel size $1.8 \mu \mathrm{m})$; (b) binarization of the Region Of Interest.

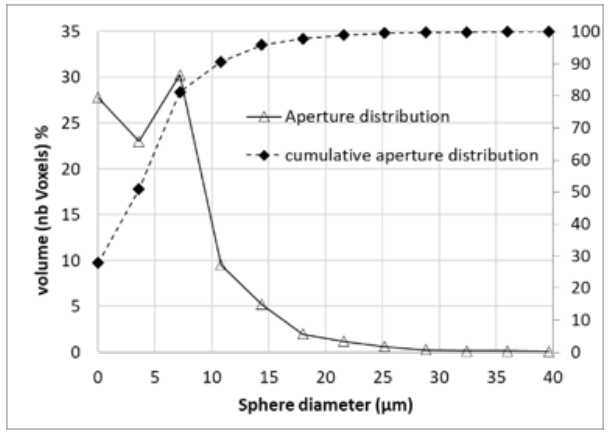

Figure 8: Aperture map distribution (65\% of the voxels belong to as sphere with diameter inferior or equal to $5 \mu \mathrm{m}$ (blue voxels)). 


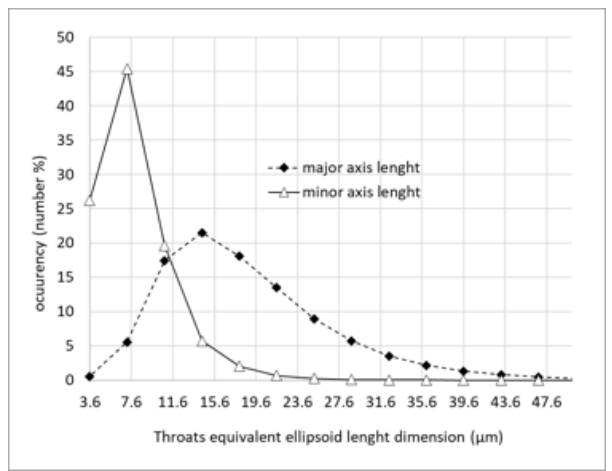

Figure 9: Throats size dimension and shape $(70 \%$ of the throats present an inner circle diameter equal to $7.6 \mu \mathrm{m}$, and $25 \%$ equal to $3.6 \mu \mathrm{m})$.

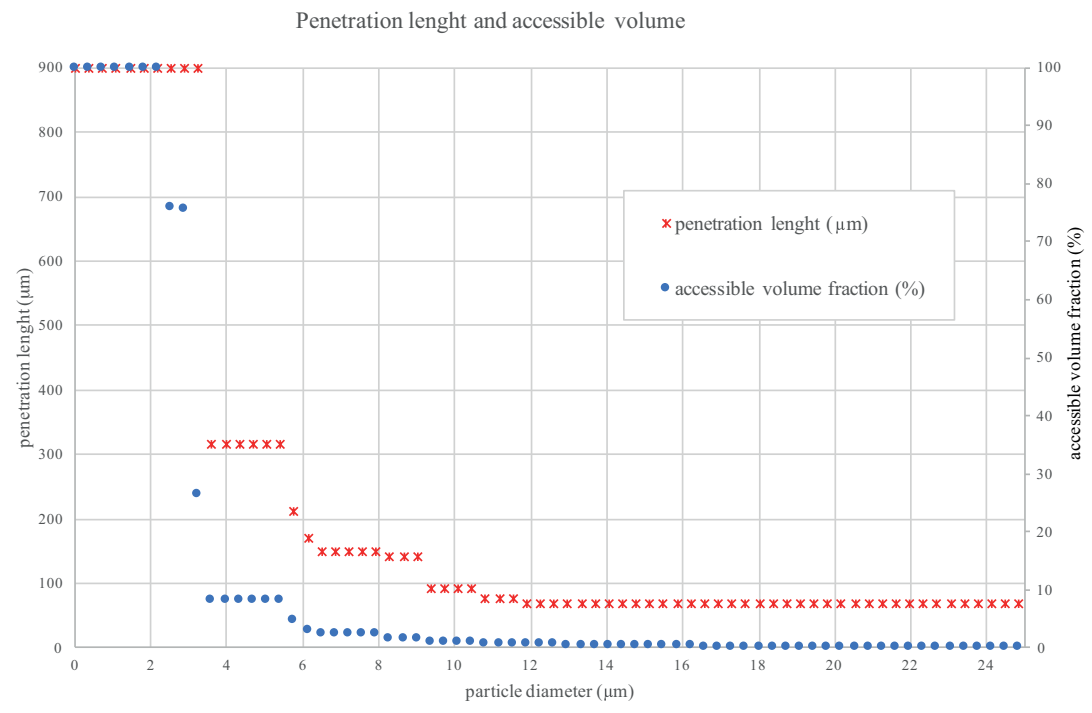

Figure 10: Microtomographic analysis: penetration length as a function of a particle diameter. 


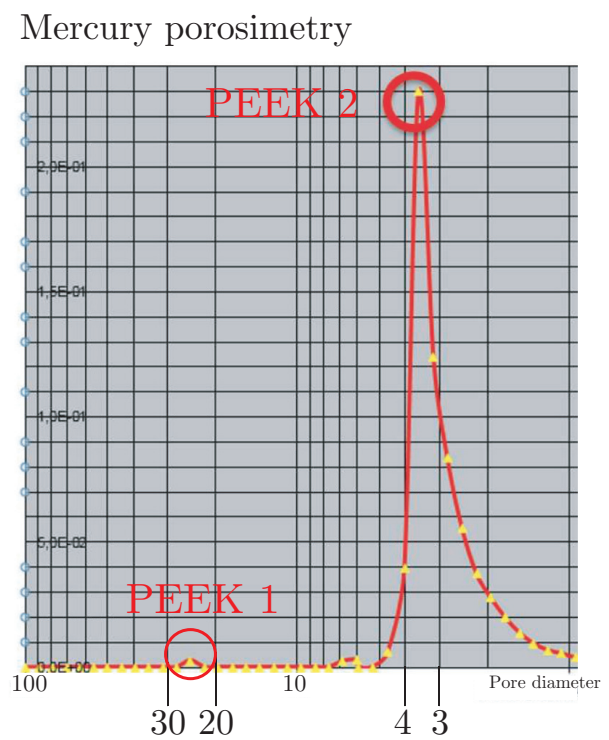

Figure 11: Mercury porosimetry. The pore size at $x$ axis is given in $\mu \mathrm{m}$. 


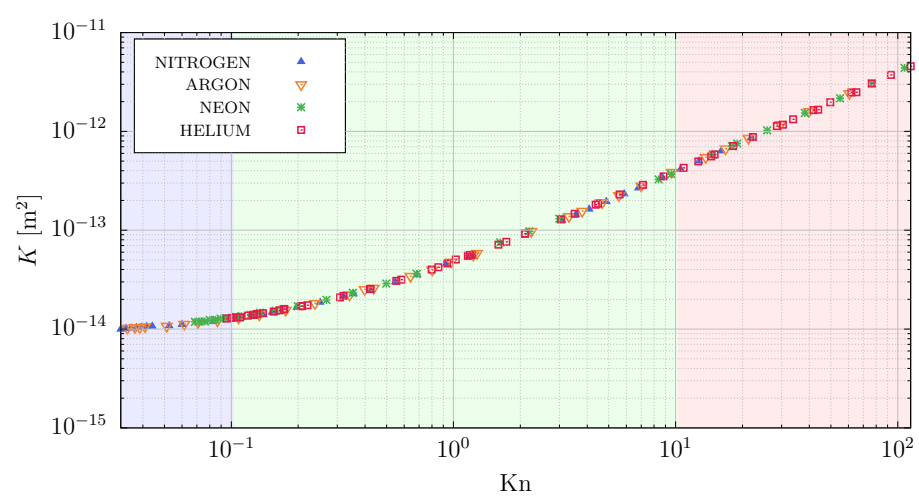

(a)

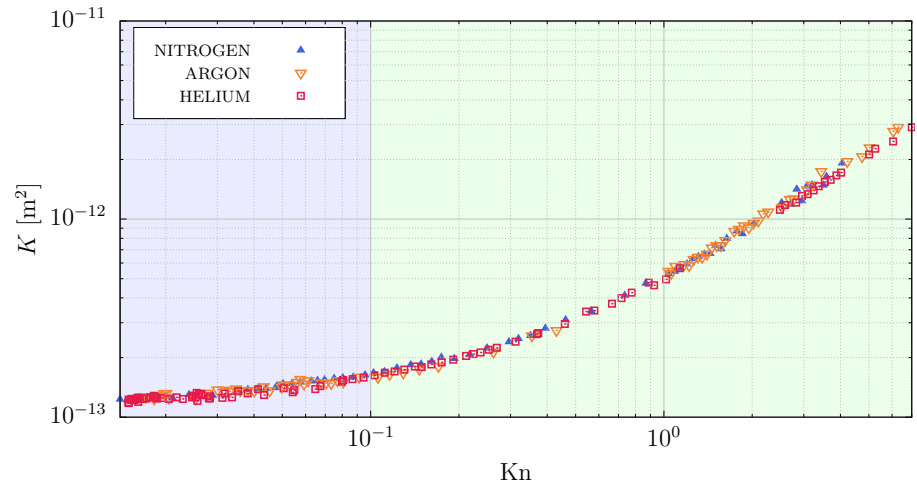

(b)

Figure 12: Permeability: (a) fist disc, (b) second disc. 


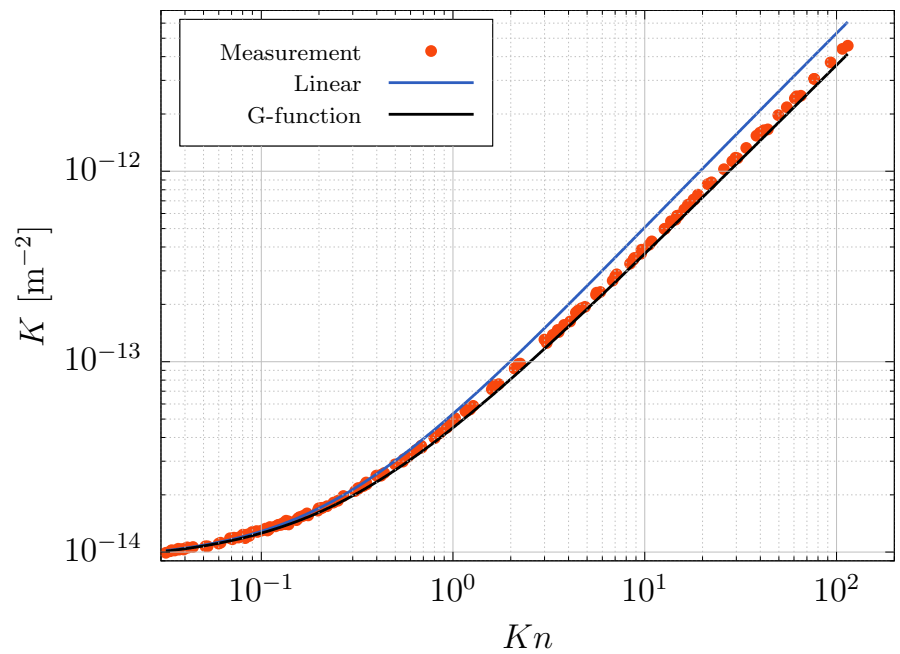

(a)

Figure 13: Measured points, presented in dimensionless form, $K / \frac{p_{\mathrm{m}} \Delta p}{\mu v_{0}^{2}} \frac{2 S}{L}$, Eq. (25), and analytical form of $K$ function, Eq. (27), (49). 


\section{AppendixA. Real gas effects}

The ideal gas law assumes that gas molecules do not occupy any space and that there is no molecular potential for attraction and repulsion. Under high pressure, the first assumption breaks down as the volume occupied by the gas molecules cannot be neglected. When the temperature is low, the molecular potential cannot be neglected, otherwise, if there is no attraction condensation cannot occur. The ideal gas law is considered accurate when the temperature is significantly larger than the boiling point, i.e. at least two times greater than the critical temperature. Additionally, the pressure needs to be not much greater than atmospheric pressure, it has to be much lower than critical pressure.

To quantify the deviation from ideal gas law conditions, it is useful to introduce the compressibility factor [39]

$$
Z=\frac{p V}{M \mathcal{R} T},
$$

which assumes unity when the ideal gas law assumption is valid. In Table A.7 the measured compressibility factors is provided for the gases used in our study and for the maximal measured pressure $(131 \mathrm{kPa})$. All the gases have the

\begin{tabular}{|c|c|}
\hline Gas & $Z$ \\
\hline \hline $\mathrm{He}$ & 1.0005 \\
\hline $\mathrm{Ne}$ & 1.0005 \\
\hline $\mathrm{N}_{2}$ & 0.9998 \\
\hline $\mathrm{Ar}$ & 0.99937 \\
\hline $\mathrm{Kr}$ & 0.99793 \\
\hline $\mathrm{Xe}$ & 0.99471 \\
\hline
\end{tabular}

Table A.7: Measured compressibility factor [39].

781

782

783

784

compressibility factor close to unity. To extend the presented mass flow rate measurement method for real gases Eq. A.1 can be used to relate the gas mass and the compressiblity factor to pressure.

\section{AppendixB. Quasi-stationary assumption}

To derive the mass flow rate we have to consider the mass variation in time as a quasi-steady process. We assume that there are infinitesimal unbalanced forces which modify the state of the system slower than the system reaches its local equilibrium. In this case, we can approximate the thermodynamic processes as a succession of equilibrium states. This approximation can be considered as an accurate one when the average time needed for a gas molecule to travel through the porous medium is much greater than the time between two successive intermolecular collisions in the reservoir [40]. To quantitatively 
estimate this time, we introduce a measure of the average time between two successive collisions, the Mean Free Time, MFT, as

$$
M F T=\ell / v_{0},
$$

where the mean free path, $\ell$, and the most probable molecular velocity, $v_{0}$, are defined by Eqs. (15) and (16), respectively. To estimate the average time for a gas molecule to travel through the porous media we introduce the Fluid Travel Time, FTT, as

$$
F T T=\frac{\ell_{\tau} L}{u},
$$

where $\ell_{\tau}$ is the tortuosity, Eq. (10), $L$ is the thickness of the porous sample and $u$ is the fluid velocity. In the hydrodynamic flow regime we have

$$
\Delta p=0.5 \rho u^{2},
$$

where $\rho$ is the gas density. Under typical experimental conditions, for the sample thickness $L=2 \mathrm{~mm}$, and assuming $\ell_{\tau}=2$, the mean free time, $M F T$, is five orders of magnitude less than the average fluid travel time, FTT, through the porous media, therefore, we are well within the quasi-stationary state assumption.

\section{AppendixC. Two limits approximation}

Additional possibility to obtain the porous medium characteristic dimension can be done by using two limits of the flow regimes, which can be easily distinguished. The first limit is the free molecular regime (or Knudsen diffusion regime), where the molecule-molecule collisions can be neglected because they are a few numerous comparing to molecule-surface collisions. In this regime, the mass flow rate is proportional to $a^{3}$. For the second limit regime, hydrodynamic regime (Poiseuille flow), the opposite situation is realized: molecule-surface collisions are very few numerous compared to molecule-molecule collisions. The mass flow rate is proportional to $a^{4}$. Therefore, we can use the ratio of the normalized mass flow rates, measured in these two regimes to find the characteristic dimension of the porous media as following

$$
\frac{\dot{M}_{\mathrm{P}} / M_{\mathrm{S} 0}}{\dot{M}_{\mathrm{FM}} / M_{\mathrm{G} 0}}=\frac{a}{4} \frac{3 \sqrt{\pi}}{8} \frac{2-\alpha}{\alpha} .
$$

However, two problems are related to the realization of this approach. First, the value of the accommodation coefficient $\alpha$ is unknown a priori. The second curtail point is the correct determination of the flow regime, i.e. the correct choice of the pressure range. From the theoretical point of view we know that the both normalized mass flow rates $\dot{M}_{\mathrm{P}} / M_{\mathrm{S} 0}$ and $\dot{M}_{\mathrm{FM}} / M_{\mathrm{G} 0}$ have to be constant. If this is not the case, the hydrodynamic (or free molecular) regime has not been reached yet, and the determination of the characteristic dimension can be affected by the essential error. Under our experimental conditions and for the porous samples used here, we did not arrive to reach both regimes, so this theoretically possible approach was not realized here. 